\title{
Resultados de la osteotomía correctora en el tratamiento de la consolidación en mala posición del radio distal
}

\author{
P. J. Delgado', R. Kaempf de Oliveira ${ }^{2}$ \\ 'Unidad de Cirugía de la Mano y del Miembro Superior. Hospital Universitario Madrid Montepríncipe. \\ Universidad San Pablo CEU. Madrid, España. \\ 2Instituto da Mâo. Complexo Hospitalar de la Santa Casa y Hospital Mâe de Deus. Porto Alegre, RS, Brasil.
}

Resumen: Introducción y objetivos: Las fracturas del tercio distal del radio consolidadas en mala posición pueden generar limitaciones funcionales y estéticas. Valoramos los resultados clínico-laborales del tratamiento quirúrgico de la consolidación en mala posición del radio distal.

Material y método: Se revisan 23 pacientes con osteotomías extraarticulares del radio distal, con un seguimiento prospectivo medio de 27 meses (rango, 12-38). La edad media era 42 años (rango, 22-62). La mano izquierda fue intervenida en 12 casos (52\%) y en $43 \%$ se trataba de la mano dominante. En 13 pacientes se realizó osteotomía de adición dorsal (57\%) y en 10 pacientes volar (43\%), en función de la deformidad ósea, a los 6 meses de media de la fractura. Se valoran: dolor según Escala Analógica Visual (EAV), rango de movilidad y parámetros radiológicos pre y postoperatorios, así como el cuestionario DASH y reincorporación laboral al final del seguimiento.

Resultados: Se obtuvo la consolidación completa en todos los casos. La EAV pasó de 7.7 a 2.5. El arco de flexo-extensión pasó de $90.6^{\circ}$ a $127.7^{\circ}$ y la rotación de $137.5^{\circ}$ a $168^{\circ}$. En el estudio radiológico, el índice radiocubital distal pasó de $+5.9 \mathrm{~mm}$ a +1.7 $\mathrm{mm}$, la inclinación radial pasó de $17^{\circ}$ a $24^{\circ}$ y la báscula volar media fue $9,5^{\circ}$. Seis pacientes con osteotomías dorsales precisaron una reintervención. Las osteotomías dorsales presentaron más ganancia de movilidad y complicaciones que las volares. No hubo diferencias respecto a dolor, parámetros radiológicos y cuestionario DASH.

Conclusiones: Los resultados laborales, mejoría del dolor, parámetros radiológicos y calidad de vida obtenido con ambas técnicas de osteotomía es similar. La osteotomía dorsal presenta mejor arco de movilidad y la volar menos complicaciones y reintervenciones.

Palabras clave: Fractura, consolidación en mala posición, radio distal, osteotomía.

\begin{abstract}
Introduction and objectives: Malunion of distal radius may produces functional and aesthetic limitations. We present clinical and functional results of surgical treatment of malunited distal radius using corrective osteotomy.

Material and Methods:We reviewed 23 patients with extra-articular osteotomy of the distal radius, with a prospective followup of 27 months (range, 12-38). The mean age was 42 years (range, 22-62). The left hand was involved in 12 cases (52\%) and $43 \%$ dominant hand. 13 patients were treated by dorsal opening wedge corrective osteotomy (57\%) and 10 patients (43\%) by volar opening wedge, as bone deformity, at 6 months after fracture. Pain (using the Visual Analogue Scale -VAS-), range of motion, X-ray parameters, DASH questionnaire (Spanish version) and time to return to work were evaluated at the end of follow up.

Results: Complete healing was obtained in all cases. The VAS increased from 7.7 to 2.5. The flexion-extension arc increased from $90.6^{\circ}$ to $127.7^{\circ}$ and rotation arc from $137.5^{\circ}$ to $168^{\circ}$. X-Ray: distal radioulnar index decreased from $+5.9 \mathrm{~mm}$ to $+1.7 \mathrm{~mm}$, radial inclination increased from $17^{\circ}$ to $24^{\circ}$ and the average volar-tilt was $9.5^{\circ}$. Six patients required a reoperation (all were dorsal osteotomies). Dorsal osteotomies had more gain mobility but more post-Op complications than volar open wedge osteotomy. No differences about pain, radiological parameters and DASH questionnaire were found.

Conclusions: Related-to-work results, pain relief, radiological parameters and functional results were similar with both techniques of osteotomy. The opening dorsal wedge osteotomy has better range of motion, more complications and reoperations than volar opening osteotomy.
\end{abstract}

Key words: Fracture, malunion, distal radius osteotomy.

La fractura distal del radio fue descrita por Colles como una patología benigna que no causaba limitaciones funcionales, apenas estéticas'. En la actualidad son varios los autores que han demostrado las posibles secuelas que pueden ocurrir cuando esta fractura no se tratacorrectamente. La consolidación en mala posi-
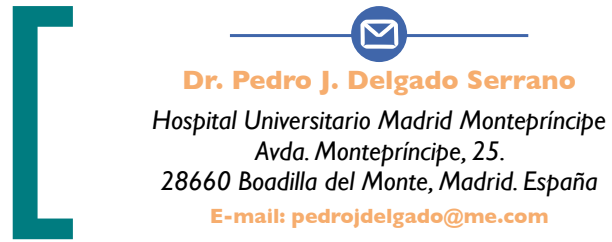

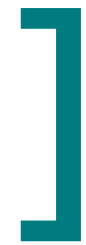


ción es la complicación más frecuente y presenta una prevalencia variable que oscila entre el 5 y el $70 \%$ de los $\operatorname{casos}^{2,3}$.

Las consolidaciones en mala posición del radio distal pueden ser divididas en extraarticulares, intraarticulares y combinadas (intra y extraarticulares) 4 . Las intraarticulares fueron determinadas por Knirk y Jupiter ${ }^{5}$ como un escalón articular de más de 2 mm, situación considerada como preartrósica. Sin embargo, en las extraarticulares la definición es más difícil y se basa en parámetros radiológicos respecto a la angulación y el acortamiento del radio distal. El amplio intervalo de prevalencia (5-80\%) de las consolidaciones en mala posición se debe a la disparidad de criterios usados para su definición. Pequeños cambios en la orientación de las superficies articulares, discrepancias de longitud y/o rotaciones no deben ser consideradas estrictamente como consolidaciones en mala posición, ya que no suelen originar deformidades relevantes ni importantes repercusiones funcionales, por lo que deben ser excluidas de esta definición ${ }^{4,6}$.

Aproximadamente el $25 \%$ de las fracturas de la extremidad distal del radio tratadas de forma ortopédica presentan un desplazamiento secundario que evolucionan a consolidaciones en mala posición ${ }^{7}$. Con el desarrollo de los sistemas de fijación, esta tasa se ha reducido hasta el 10\%, si bien el $80 \%$ de las osteotomías correctoras se realizan en fracturas que fueron tratadas inicialmente de forma conservadora ${ }^{6,8}$.

La consolidación en mala posición de fracturas de la extremidad distal del radio altera la función normal de las articulaciones radiocarpiana y radiocubital distal'. El acortamiento y la pérdida de la báscula volar del radio distal originan pérdida de fuerza, disminución de la movilidad, deformidad y dolor ${ }^{10}$. Una pérdida de la inclinación radial superior a $20^{\circ}$ en el plano sagital, $10^{\circ}$ en el coronal, rotación mayor de $10^{\circ}$ o un acortamiento superior a $4 \mathrm{~mm}$ suelen producir alteraciones sintomáticas, por lo que está indicado su corrección de forma quirúrgica"l.

El objetivo de la osteotomía es corregir la orientación de la muñeca permitiendo, por el realineamiento óseo, una distribución homogénea de las fuerzas y una cinemática normal de las articulaciones radiocarpiana, medio-carpiana y radiocubital distal.

Los resultados de las osteotomías distales del radio por consolidación en mala posición son mejores (80\% de buenos y excelentes) en pacientes con deformidad extraarticular, buena movilidad preoperatoria (70\% de movilidad comparado con el lado sano), sin cambios degenerativos articulares con buena alineación mediocarpiana y acortamiento radial inferior a $10 \mathrm{~mm}^{4}$.

La osteotomía del radio distal está contraindicada en pacientes con poca sintomatología a pesar de presentar alteraciones radiográficas importantes. Estos pacientes generalmente suelen ser mayores y con baja demanda funcional. Tampoco está indicada en pacientes con inestabilidad mediocarpiana irreductible, artrosis mediocarpiana y signos de síndrome doloroso regional complejo ${ }^{4}$.

Existen diversos trabajos publicados sobre el resultado clínico y radiológico del tratamiento quirúrgico de esta deformidad, pero son series muy heterogéneas respecto a sexo y edad, con resultados poco centrados en la repercusión funcional e impacto labora| $\left.\right|^{8,11}$.

El objetivo del estudio ha sido la valoración de los resultados clínico-laborales del tratamiento quirúrgico de la consolidación en mala posición del radio distal mediante osteotomía extraarticular dorsal y/o volar, en función de la deformidad radiológica, en pacientes jóvenes y edad laboral activa.

\section{MATERIAL Y MÉTODO}

Estudio prospectivo de 23 pacientes que presentaban una consolidación en mala posición del radio distal tratados quirúrgicamente por el autor principal (PJD) en el Hospital FREMAP de Majadahonda (Madrid, España). Se realiza un estudio prospectivo con un seguimiento medio de 27 meses (rango, 12-38).

La clasificación de las fracturas se basó en la propuesta por Fernández y Júpiter a partir de los exámenes radiológicos (proyecciones anteroposterior y lateral de muñeca), siendo en nuestro grupo de tipo I (fractura de la metáfisis extraarticular) ${ }^{12}$.

Las indicaciones para la osteotomía fueron dolor (más de 3 puntos en estático y más de 5 en dinámico), pérdida de movilidad, parámetros radiológicos no aceptables (pérdida de la inclinación del radio mayor de $20^{\circ}$ en el plano sagital, $10^{\circ}$ en el coronal, rotación de más de $10^{\circ}$ y acortamiento superior a $4 \mathrm{~mm}$ ), limitación funcional, pérdida de movilidad e incapacidad para retornar a su actividad laboral previa. Se consideraron criterios de exclusión aquellos parámetros radiológicos de deformidad aceptables (pérdida de la inclinación del 
radio menor o igual a $20^{\circ}$ en el plano sagital, menor o igual a $10^{\circ}$ en el coronal, rotación de menos de $10^{\circ}$ y acortamiento igual o menor a $3 \mathrm{~mm}$ ), cambios degenerativos avanzados, osteopenia importante y signos clínicos de síndrome doloroso regional complejo. Para la elaboración del presente estudio, fueron excluidos aquellos pacientes con seguimiento inferior a 12 meses, con técnicas de fijación distintas a la placa dorsal o volar descritas en el apartado de "técnica quirúrgica", pacientes sin resultado laboral definitivo y estudio radiográfico o evolutivo completos.

Dieciséis pacientes eran hombres y siete eran mujeres, con una edad media de 42 años (rango, 22-62 años) y actividades laborales de baja (13\%), media (35\%) y alta demanda funcional (52\%). La mano izquierda fue intervenida en 12 casos (52\%) y en $43 \%$ se trataba de la mano dominante@ Tabla I.

El tratamiento inicial fue ortopédico, mediante reducción cerrada e inmovilización con yeso antebraquial, en 18 pacientes (78\%). Los 5 pacientes restantes fueron intervenidos previamente: 4 mediante reducción cerrada y fijación percutánea con agujas de Kirschner y un paciente mediante reducción cerrada y fijación externa.

El tiempo transcurrido desde la fractura de la extremidad distal del radio y la osteotomía correctora fueron I 85 días (rango, 92-320 dias). Todos los pacientes presentaban dolor que limitaba su función, de 7.7 puntos de media (rango, 4-9.I puntos) en la escala analógico visual de $0-10$.

En todos los casos se objetivó una pérdida del rango de movilidad activa, con una movilidad media de $41.7^{\circ}$ de flexión, 48.9 de extensión, $18.6^{\circ}$ de flexión radial, $22.5^{\circ}$ de flexión cubital, $81^{\circ}$ de pronación y $56.4^{\circ}$ de supinación.

La planificación pre-operatoria se realizó a partir de radiografías comparadas con la muñeca contralateral. Se utilizaron proyecciones estándar anteroposterior y lateral de muñeca, midiendo los parámetros según la modificación de Castaing ${ }^{13}$ del método descrito por Gartland y Werley ${ }^{14}$. La inclinación radial se midió en grados, como el ángulo que forma la articulación radiocarpiana respecto a la línea perpendicular al eje de la diáfisis del radio. La varianza cubital se define, en milímetros, como la relación entre la superficie articular del radio y la de la cabeza del radio, determinada como la distancia entre la línea perpendicular al eje de la diáfisis del radio tangencial a la carilla articular semilunar del radio y la superficie articular distal del radio. La báscula radial se mide en grados como el ángulo entre la línea tangencial de la superficie articular del radio en el plano sagital respecto al eje longitudinal del radio. Se objetivó una inclinación radial de $17^{\circ}$ (rango, - $\left.14-30^{\circ}\right)$ y un índice radiocubital distal de $+5.8 \mathrm{~mm}$ (rango, 0-10). Trece pacientes (57\%) presentaban una báscula dorsal de $18.8^{\circ}$ de media (rango, $3-25^{\circ}$ ) y 10 pacientes (43\%) una báscula volar de $24.1^{\circ}$ de media (rango, $10-40^{\circ}$ ) en el estudio radiológico preoperatorio (-) Tabla $\mathrm{I}$.

Todos los pacientes del estudio fueron valorados como accidente de trabajo, según la definición de la Ley de Seguridad Social de 21 de abril de 1966 y Texto Refundido de la Ley General de la Seguridad Social de 30 de mayo de 1974, artículo 84 que dice textualmente: "Se entiende por accidente de trabajo toda lesión corporal que el trabajador sufra con ocasión o por consecuencia del trabajo que ejecute por cuenta ajena"'15.

\section{TÉCNICA QUIRÚRGICA}

La técnica quirúrgica varió en función de la deformidad en el plano sagital @ Figura I.

En los casos con deformidad dorsal del radio (13 pacientes), se realizó una osteotomía de adición a través de un abordaje clásico entre el tercer y cuarto compartimentos dorsales de la muñeca. Se utilizó un injerto en cuña tricortical procedente de la cresta iliaca contralateral y chips de esponjosa compactada, según técnica de Fernández ${ }^{4}$. Para la fijación se utilizaron 2 placas de bajo perfil del Profile Hand Standard Plate (Stryker Leibinger, Friburgo, Alemania) de $2.3 \mathrm{~mm}$ paralelas al eje del radio en $1 \mathrm{I}$ casos y agujas de Kirschner percutáneas de $1.8 \mathrm{~mm}$ en 2 casos (4) Figuras I, $2,3,4$ y 5 .

Los pacientes que presentaban deformidad volar ( 10 pacientes), se realizó una osteotomía de adición con injerto procedente de cresta iliaca contralateral a través de una vía de Henry convencional (1) Figuras 6, 7 , y 8 , según técnica descrita por Shea et $a^{16}$. En todos los casos se utilizó para la fijación una placa de bajo perfil adaptada modelos Matrix - SmartLock $2.7 \mathrm{~mm}$ (Stryker Leibinger, Friburgo, Alemania) y LCP Distal Radius Plate 2.4 mm (Synthes, Paoli, Pennsylvania, EEUU). En un caso fue necesario utilizar una aguja de Kirschner percutánea a través de la estiloides radial, como método de fijación complementario temporal. 


\section{TABLA I - DATOS PREOPERATORIOS DE LOS PACIENTES.}

\begin{tabular}{|c|c|c|c|c|c|c|c|c|c|c|c|c|c|c|c|c|}
\hline \multicolumn{6}{|c|}{ Datos del paciente } & \multirow{2}{*}{ EVA } & \multicolumn{6}{|c|}{ Rango de movilidad } & \multicolumn{4}{|c|}{ Parámetros radiológicos } \\
\hline Caso & Edad & Sexo & Lado & Dom. & Trabajo & & FLX & EXT & $\mathrm{DC}$ & $\mathrm{DR}$ & PR & SUP & IRCD & $\mathbb{R}$ & Báscula & $\mathrm{BR}$ \\
\hline 1 & 38 & $\mathrm{H}$ & I & No & Cerrajero & 8,1 & 48 & 40 & 22 & 24 & 70 & 36 & 8 & 16 & Dorsal & 16 \\
\hline 2 & 32 & $\mathrm{H}$ & 1 & No & Construcción & 8,1 & 50 & 55 & 30 & 20 & 90 & 30 & 9 & 28 & Volar & 40 \\
\hline 3 & 56 & $\mathrm{H}$ & D & $\mathrm{Si}$ & Comercial & 7,9 & 45 & 38 & 20 & 23 & 68 & 32 & 8 & 25 & Dorsal & 15 \\
\hline 4 & 47 & M & D & $\mathrm{Si}$ & Reponedora & 7,5 & 60 & 30 & 20 & 25 & 90 & 40 & 2 & 15 & Volar & 25 \\
\hline 5 & 58 & $\mathrm{H}$ & D & $\mathrm{Si}$ & Especialista & 7,0 & 50 & 30 & 15 & 25 & 90 & 90 & 6 & -14 & Dorsal & 3 \\
\hline 6 & 45 & $M$ & D & $\mathrm{Si}$ & Limpieza & 7,1 & 50 & 50 & 20 & 30 & 90 & 90 & 6 & 2 & Dorsal & 5 \\
\hline 7 & 34 & $\mathrm{H}$ & I & No & Montaje & 7,1 & 45 & 95 & 20 & 10 & 85 & 90 & 5 & 15 & Dorsal & 20 \\
\hline 8 & 24 & M & I & No & Auxiliar sanitario & 8,1 & 60 & 60 & 40 & 20 & 90 & 70 & 1 & 24 & Dorsal & 22 \\
\hline 9 & 41 & M & D & $\mathrm{Si}$ & Montaje & 7,0 & 30 & 40 & 10 & 15 & 90 & 90 & 4 & 22 & Dorsal & 20 \\
\hline 10 & 50 & $\mathrm{H}$ & I & No & Construcción & 8,1 & 35 & 50 & 30 & 20 & 90 & 30 & 6 & 30 & Dorsal & 22 \\
\hline 11 & 48 & M & I & No & Limpieza & 9,1 & 25 & 60 & 15 & 10 & 90 & 60 & 6 & 15 & Dorsal & 15 \\
\hline 12 & 41 & $\mathrm{H}$ & 1 & No & Electricista & 9,1 & 50 & 45 & 10 & 25 & 90 & 65 & 10 & 10 & Volar & 20 \\
\hline 13 & 61 & $\mathrm{H}$ & D & $\mathrm{Si}$ & Construcción & 9,1 & 40 & 45 & 35 & 15 & 85 & 90 & 0 & 15 & Volar & 20 \\
\hline 14 & 47 & $\mathrm{H}$ & D & $\mathrm{Si}$ & Construcción & 8,1 & 30 & 45 & 24 & 12 & 85 & 45 & 10 & 15 & Volar & 25 \\
\hline 15 & 62 & M & I & No & Limpieza & 8,1 & 30 & 60 & 22 & 18 & 90 & 50 & 9 & 14 & Volar & 10 \\
\hline 16 & 24 & $\mathrm{H}$ & $D$ & No & Montaje & 7,0 & 30 & 50 & 35 & 16 & 90 & 50 & 8 & 30 & Volar & 40 \\
\hline 17 & 41 & $\mathrm{H}$ & I & No & Montaje & 3 & 35 & 40 & 40 & 15 & 80 & 50 & 6 & 18 & Dorsal & 22 \\
\hline 18 & 22 & $\mathrm{H}$ & D & $\mathrm{Si}$ & Construcción & 8 & 35 & 25 & 20 & 10 & 30 & 30 & 3 & 15 & Dorsal & 20 \\
\hline 19 & 41 & M & I & No & Auxiliar sanitario & 8,1 & 20 & 30 & 10 & 15 & 40 & 20 & 4 & 24 & Dorsal & 10 \\
\hline 20 & 33 & $\mathrm{H}$ & D & $\mathrm{Si}$ & Montaje & 6,1 & 70 & 80 & 15 & 20 & 80 & 80 & 7 & 20 & Volar & 22 \\
\hline 21 & 54 & $\mathrm{H}$ & I & No & Construcción & 9,1 & 35 & 70 & 20 & 20 & 85 & 30 & 8 & 20 & Dorsal & 18 \\
\hline 22 & 40 & $\mathrm{H}$ & $D$ & $\mathrm{Si}$ & Construcción & 8,2 & 45 & 50 & 25 & 15 & 80 & 70 & 6 & 18 & Volar & 15 \\
\hline 23 & 32 & $\mathrm{H}$ & I & No & Pintor & 8,8 & 40 & 45 & 20 & 24 & 85 & 60 & 5 & 15 & Volar & 18 \\
\hline
\end{tabular}

Datos del paciente: H: hombre, M: mujer, l: izquierdo, D: derecho. La edad se expresa en años. EVA: Escala Visual Analógica (dolor). Rango de movilidad (se expresan en grados), FLX: flexión, EXT: extensión, DC: desplazamiento cubital, DR: desplazamiento radial, PR: pronación, SUP: supinación. Parámetros radiológicos: IRCD: índice radiocubital distal (se expresa en milímetros), IR: inclinación radial, BR: báscula radial (estos dos últimos se expresan en grados).

Tras la cirugía se inmovilizó la muñeca con una férula antebraquial volar en posición neutra dejando libre las articulaciones metacarpofalángicas para su movilización inmediata.

Todos los procedimientos utilizados en el tratamiento fueron realizados tras la obtención de un consentimiento informado por parte del paciente. En el postoperatorio recibieron profilaxis antibiótica mediante
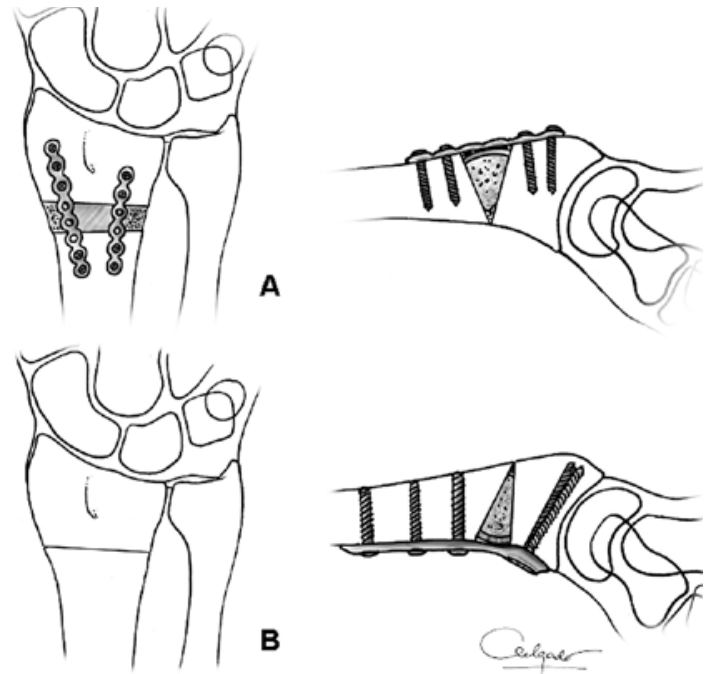

Figura I. Técnica quirúrgica: osteotomía correctora de adición dorsal A. y volar B. 


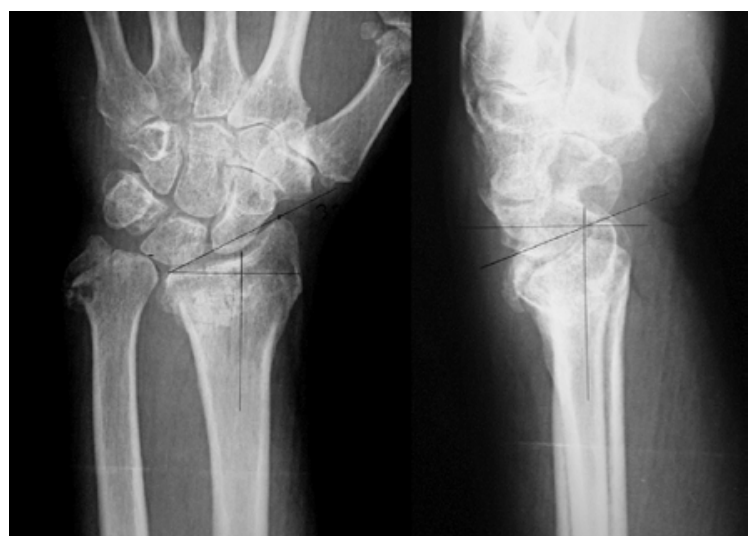

Figura. 2. Imagen radiológica donde se aprecia una consolidación en mala posición con acortamiento y báscula dorsal del radio respecto al cúbito y carpo.

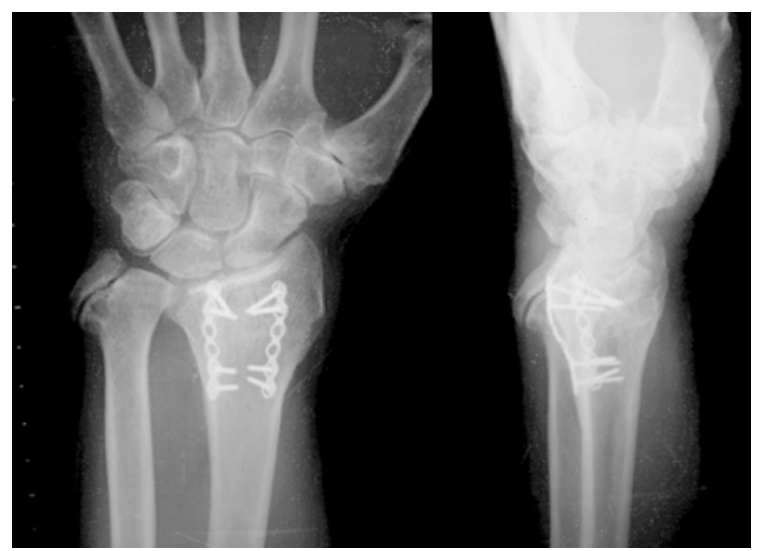

Figura 4. Control radiológico de la osteotomía de adición dorsal.

cefazolina 2 g cada 8 horas, IV por 24 horas o clindamicina $600 \mathrm{mg}$ cada 12 horas, IV por 24 horas en caso de alergia a penicilina o derivados, según protocolo de la comisión de infecciones del centro.

No se utilizaron sustitutivos óseos ni se realizaron gestos adicionales a nivel de la articulación radiocubital distal. Con el objetivo de disminuir la inflamación pre$\mathrm{coz}$, en el postoperatorio inmediato se administraron $250 \mathrm{mg}$ de metilprednisolona (Urbason $囚$ ) diluidos en 100 cc I. V. en dosis única y se mantuvo el miembro en elevación por 24-48 horas, tras el cual se dió alta hospitalaria, para seguimiento por consultas externas.

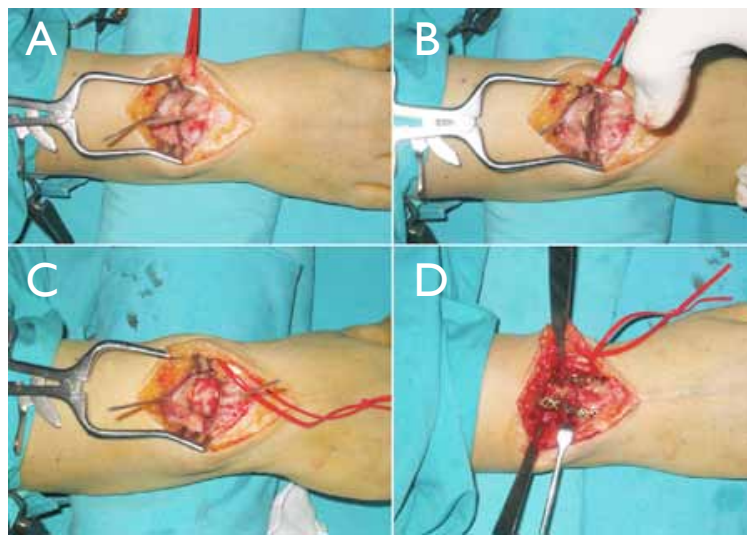

Figura 3. Imagen intraoperatoria de la osteotomía dorsal correctora. Colocación de agujas-guía y osteotomía A. y B. Aporte de injerto de cresta iliaca y fijación con placas de bajo perfil C. y D.

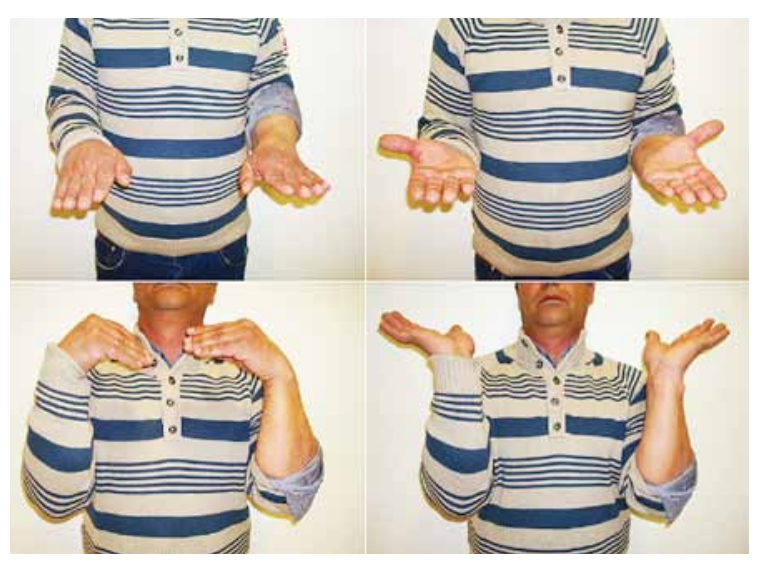

Figura 5. Resultado clínico a los 2 años de la intervención.

\section{TRATAMIENTO POSTOPERATORIO}

A las 4 semanas se retira la inmovilización y se inicia la movilización activa y pasiva de la muñeca de forma suave. A las 6 semanas se intensifican las medidas antiedema y antiinflamatorias y se inicia la cinesiterapia activa y pasiva de forma gradual y progresiva, según protocolo de rehabilitación del centro, hasta el alta laboral, por un mínimo de 6 semanas.

\section{EVALUACIÓN DE LOS RESULTADOS}

El análisis de los resultados se realiza desde el punto de vista funcional, radiológico y laboral al alta laboral y al final del seguimiento. 
Los resultados funcionales valoran el rango de movilidad activa, escala visual analógica del dolor (EVA) y puntuación de la versión estandar validada en español del cuestionario DASH'17.

Los parámetros valorados en el estudio radiológico son la consolidación ósea, el índice radiocubital distal, ángulo de inclinación radial, ángulo de inclinación volar, escalón articular y signos degenerativos al final del seguimiento.

El resultado laboral se analizó valorando los días de baja totales desde la cirugía y la medición de las secuelas e incorporación a su trabajo, siguiendo la escala de valoración del Instituto Nacional de la Salud (INS) que determina las categorías de curado, secuelas con baremo (no presentan secuelas que originen un grado de incapacidad), incapacidad permanente parcial (el paciente puede realizar su trabajo pero con restricciones inferiores al 33\% de la actividad), incapacidad permanente total (imposibilidad de realizar su trabajo previo) y grandes incapacidades (incapacidad absoluta y gran invalidez $)^{15}$.

El análisis estadístico de los resultados se llevó a cabo con medidas de centralización y descentralización y el análisis de la varianza de una vía con el paquete estadístico SPSS. Se consideró que existían diferencias estadísticamente significativas cuando $p<0,05$.

\section{RESULTADOS}

Todos los pacientes presentaron mejoría del dolor, que mejoró una media de 5.3 puntos en la EAV, pasando de 7.7 antes de la cirugía a 2.6 puntos al final del seguimiento. No se encontraron diferencias de mejoría del dolor respecto a la técnica de osteotomía (2) Figura 2.

El rango de movilidad se incrementó en todos los arcos. La flexión media pasó de $41.7^{\circ}$ (rango, 20-80) a $62.2^{\circ}$ (rango, $40-80^{\circ}$ ) y la extensión media de $26.5^{\circ}$ $\left(30-90^{\circ}\right)$ a $65.2^{\circ}$ (rango, 35-90 $)$. La desviación cubital se incrementó de $22.5^{\circ}$ (rango, $0-50^{\circ}$ ) a $32.7^{\circ}$ (rango, $25-40^{\circ}$ ) y la radial de $18.6^{\circ}\left(\right.$ rango, $\left.0-30^{\circ}\right)$ a $23.7^{\circ}$ (rango, $\left.25-40^{\circ}\right)$. El arco de rotación pasó de $81.1^{\circ}$ de pronación media (rango, $30-90^{\circ}$ ) a $86.8^{\circ}$ (rango, $85-90^{\circ}$ ) y la supinación media se incrementó de $56.4^{\circ}$ (rango, $20-90^{\circ}$ ) a $84.5^{\circ}$ (rango, $55-90^{\circ}$ ).

Los pacientes tratados con osteotomía de adición dorsal presentaron un incremento de movilidad mayor en los arcos de flexo-extensión y desviación lateral.
Aquellos tratados con osteotomía volar presentaron una ganancia de movilidad mayor en el arco de rotación del antebrazo, especialmente en la supinación.

Se obtuvo la consolidación completa en todos los casos. En el estudio radiológico, la inclinación radial pasó de $17^{\circ}$ (rango, $\left.-14-30^{\circ}\right)$ a $24^{\circ}$ (rango, $16-30^{\circ}$ ) y el índice radiocubital distal pasó de $+5.8 \mathrm{~mm}$ (rango, 0 - 10 $\mathrm{mm}$ ) a $1.7 \mathrm{~mm}$ (rango, 0-6 mm).

La báscula radial media postoperatoria fue $9.9^{\circ}$ volares (rango, $2-20^{\circ}$ ). Los 13 pacientes con báscula dorsal, pasaron de $18.8^{\circ}$ dorsales a $9.5^{\circ}$ volares y en los 10 pacientes con báscula volar de $24.1^{\circ}$ pasaron a $10.4^{\circ}$ al final del tratamiento. No se encontraron diferencias respecto al tipo de osteotomía realizada respecto a los parámetros radiológicos, ni aparición de signos degenerativos al final del seguimiento (a) Figura 3.

La puntuación media en el cuestionario DASH al final del seguimiento fueron 21 puntos (rango, 0-44). Se encontró un discreto mejor resultado en los casos tratados con osteotomía dorsal (16.9) respecto a la dorsal (24.6).

Los pacientes volvieron a trabajar a los 125 días de media (rango, 58-380 dias) de la osteotomía. Las osteotomías volares presentaban menor número de días de baja laboral (94) con respecto a las dorsales (I 48.5).

Todos los pacientes retornaron a su mismo puesto de trabajo previo, sin diferencias respecto al nivel de demanda funcional y el tipo de deformidad previa a la intervención. Cinco pacientes (20\%) presentaban limitaciones que justificaron una incapacidad permanente parcial tras su paso por el tribunal de valoración de incapacidades.

Aparecieron complicaciones en 12 pacientes (52\%), siendo superior en los casos tratados mediante osteotomía dorsal (56\%) respecto a los tratados con osteotomía volar (30\%). 6 pacientes que fueron intervenidos mediante osteotomías dorsales precisaron una reintervención: en 5 casos se precisó retirar el material de fijación y un paciente precisó una artroplastia de resección de Darrach @ Tabla 4.

\section{DISCUSIÓN}

La deformidad del radio distal puede originar alteraciones de la biomecánica del carpo y de la articulación radiocarpiana. Cuando existe una angulación dorsal en 


\section{TABLA II - EVALUACIÓN FUNCIONAL Y RADIOLÓGICA DE LOS PACIENTES TRAS LA OSTEOTOMÍA CORRECTORA.}

\begin{tabular}{|c|c|c|c|c|c|c|c|c|c|c|c|c|c|c|c|c|}
\hline \multirow{2}{*}{ Caso } & \multirow{2}{*}{ EVA } & \multirow{2}{*}{ DASH } & \multicolumn{6}{|c|}{ Rango de movilidad } & \multicolumn{4}{|c|}{ Parámetros radiológicos } & \multirow{2}{*}{ Complicaciones } & \multirow{2}{*}{$\begin{array}{l}\text { Reinterven- } \\
\text { ciones }\end{array}$} & \multicolumn{2}{|c|}{ Resultado laboral } \\
\hline & & & FLX & EXT & $D C$ & DR & PR & SUP & IRCD & $\mathbb{R}$ & Báscula & $B R$ & & & Alta (días) & Reincorporación \\
\hline 1 & 2 & 22 & 62 & 45 & 25 & 23 & 90 & 90 & 0 & 22 & Dorsal & 9 & No & No & 77 & Completa \\
\hline 2 & 2,1 & 13,3 & 60 & 70 & 28 & 30 & 90 & 90 & 0 & 20 & Volar & 10 & STC & No & 114 & Completa \\
\hline 3 & 2 & 0,93 & 55 & 50 & 40 & 20 & 90 & 90 & 0 & 25 & Dorsal & 8 & No & No & 74 & Completa \\
\hline 4 & 5,1 & 58 & 80 & 70 & 30 & 20 & 80 & 75 & 0 & 27 & Volar & 2 & DSR & No & 100 & Completa \\
\hline 5 & 3,1 & 49 & 20 & 35 & 0 & 30 & 45 & 45 & 1 & 16 & Dorsal & 3 & Sección parcial EPL & EMO & 350 & Completa \\
\hline 6 & 2,1 & 0 & 60 & 60 & 40 & 30 & 90 & 90 & 6 & 18 & Dorsal & 20 & No & No & 93 & Completa \\
\hline 7 & 2,1 & 12 & 70 & 90 & 30 & 20 & 90 & 90 & 2 & 30 & Dorsal & 8 & Sinovitis dorsal & EMO & 81 & Completa \\
\hline 8 & 1,1 & 15 & 80 & 80 & 30 & 25 & 90 & 90 & 0 & 30 & Dorsal & 10 & STC & No & 64 & Completa \\
\hline 9 & 3 & 22 & 40 & 30 & 20 & 20 & 90 & 90 & 2 & 22 & Dorsal & 10 & Sinovitis dorsal & No & 180 & Parcial \\
\hline 10 & 1,5 & 13,5 & 60 & 60 & 40 & 40 & 90 & 80 & 6 & 30 & Dorsal & 10 & No & No & 114 & Completa \\
\hline 11 & 4,1 & 35 & 50 & 65 & 25 & 20 & 90 & 80 & 2 & 30 & Dorsal & 7 & No & No & 90 & Parcial \\
\hline 12 & 2,1 & 18 & 60 & 90 & 30 & 25 & 90 & 70 & 2 & 25 & Volar & 8 & No & No & 58 & Curado \\
\hline 13 & 5,1 & 40 & 50 & 60 & 20 & 18 & 90 & 90 & 0 & 25 & Volar & 8 & No & No & 90 & Completa \\
\hline 14 & 3,3 & 43,1 & 40 & 50 & 30 & 20 & 85 & 90 & 3 & 22 & Volar & 10 & No & No & 75 & Completa \\
\hline 15 & 3 & 12 & 80 & 80 & 40 & 30 & 90 & 90 & 4 & 25 & Volar & 20 & No & No & 130 & Completa \\
\hline 16 & 4 & 17 & 70 & 70 & 10 & 10 & 90 & 70 & 2 & 30 & Volar & 10 & No & No & 150 & Completa \\
\hline 17 & 3 & 25 & 50 & 40 & 50 & 20 & 90 & 90 & 1 & 28 & Dorsal & 8 & No & Darrach & 200 & Parcial \\
\hline 18 & 2 & 29,6 & 75 & 75 & 25 & 30 & 90 & 85 & 0 & 25 & Dorsal & 10 & Tumefacción post-op & EMO & 244 & Parcial \\
\hline 19 & 2 & 25 & 45 & 70 & 30 & 20 & 90 & 55 & 0 & 25 & Dorsal & 10 & Disestesias 3er dedo & EMO & 129 & Completa \\
\hline 20 & 0 & 0 & 75 & 85 & 30 & 20 & 90 & 90 & 0 & 18 & Volar & 9 & Disestesias en pulgar & No & 60 & Completa \\
\hline 21 & 2,1 & 8 & 55 & 40 & 25 & 15 & 90 & 90 & 2 & 25 & Dorsal & 8 & No & No & 157 & Completa \\
\hline 22 & 2 & 10 & 80 & 80 & 40 & 30 & 80 & 90 & 1 & 18 & Volar & 12 & No & No & 100 & Completa \\
\hline 23 & 3 & 15 & 80 & 90 & 40 & 28 & 85 & 90 & 5 & 15 & Volar & 18 & No & No & 145 & Completa \\
\hline
\end{tabular}

EVA: Escala Visual Analógica (dolor). Rango de movilidad (se expresan en grados), FLX: flexión, EXT: extensión, DC: desplazamiento cubital, DR: desplazamiento radial, PR: pronación, SUP: supinación. Parámetros radiológicos: IRCD: índice radiocubital distal (se expresa en milímetros), IR: inclinación radial, BR: báscula radial (estos dos últimos se expresan en grados).

TABLA III - CoMparación DE LOS RESULTADOS CLÍNICOS Y RADIOLÓGICOS TRAS LA OSTEOTOMÍA CORRECTORA DEL RADIAL DISTAL.

PRE-OPERATORIO | POST-OPERATORIO | P

\section{Dolor}

$8(7.1-8.1)$

$2.1(2.0-3.1)$

$<0.01$

\section{Rango de movilidad}

\begin{tabular}{|cccc}
\hline Flexión $\left(^{\circ}\right)$ & $40(30-50)$ & $60(70-75)$ & $<0.01$ \\
\hline Extensión $\left(^{\circ}\right)$ & $45(40-60)$ & $70(50-80)$ & 0.001 \\
\hline Desviación cubital $\left({ }^{\circ}\right)$ & $20(15-30)$ & $30(25-40)$ & N. S. \\
\hline Desviación radial $\left(^{\circ}\right)$ & $20(15-24)$ & $20(20-30)$ & 0.006 \\
\hline Pronación $\left(^{\circ}\right)$ & $85(80-90)$ & $90(90-90)$ & N. S. \\
\hline Supinación $\left(^{\circ}\right)$ & $50(32-80)$ & $90(80-90)$ & 0.001 \\
\hline Parámetros radiológicos & & & \\
\hline $\operatorname{IRCD}(\mathrm{mm})$ & $6(4-8)$ & $1(0-2)$ & $<0.01$ \\
\hline $\operatorname{RR}\left({ }^{\circ}\right)$ & $16(15-24)$ & $25(20-28)$ & 0.001 \\
\hline $\operatorname{BR}\left({ }^{\circ}\right)$ & $20(15-22)$ & $10(8-10)$ & 0.002 \\
\hline
\end{tabular}

EVA: Escala Visual Analógica (dolor). Parámetros radiológicos: IRCD índice radiocubital distal, IR: inclinación radial, BR: báscula radial. N. S.: no significativo. 


\section{TABIA IV - COMPLICACIONES ENCONTRADAS AL FINAL DEL SEGUIMIENTO EN FUNCIÓN DEL TIPO DE OSTEOTOMÍA (EXPRESADOS EN \% EN FUNCIÓN DEL TOTAL DE PACIENTES DE CADA GRUPO).}

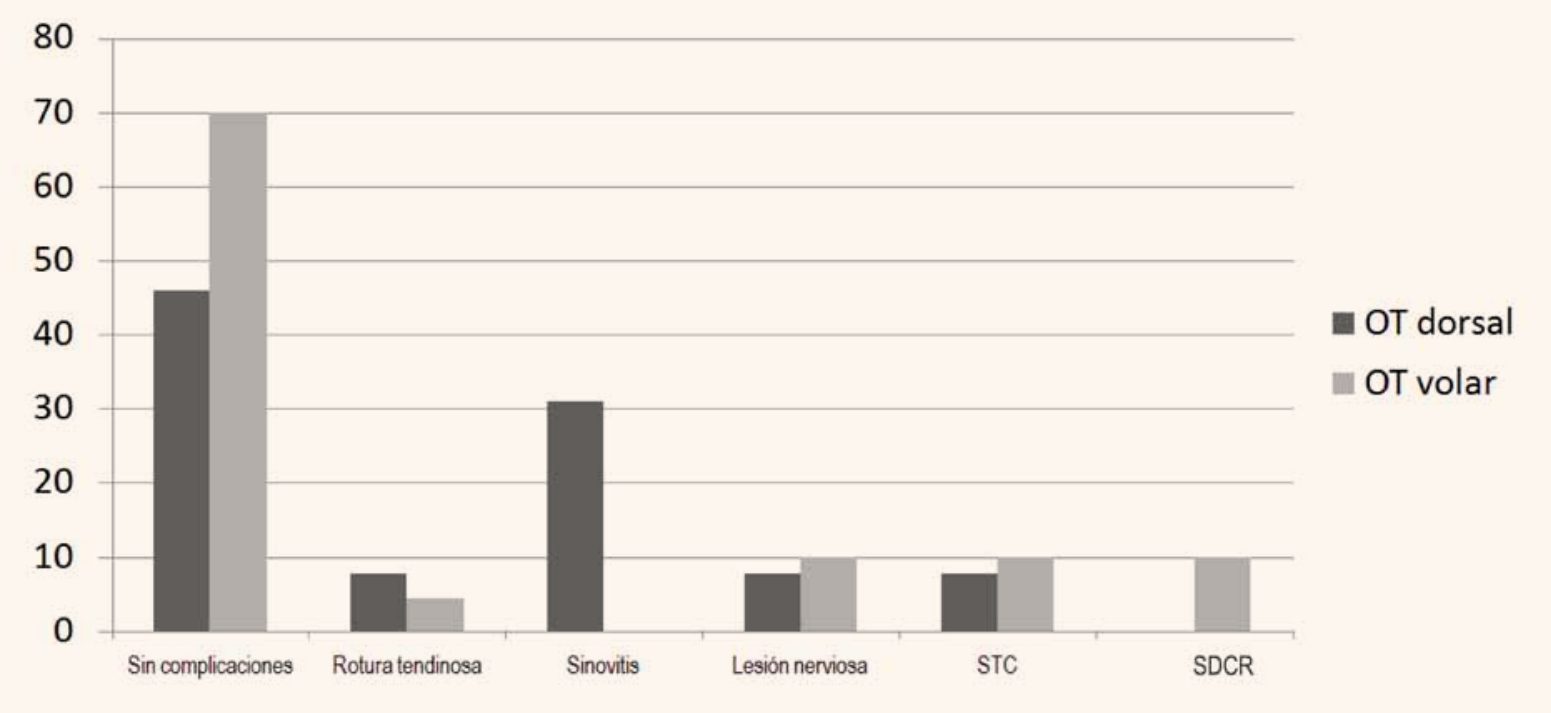

OT: Osteotomía, STC: Síndrome del túnel carpiano, SDCR: Síndrome de dolor complejo regional.

el plano sagital superior a $20^{\circ}$, las superficies de contacto y los ejes de carga sufren una traslación dorsal, originando una subluxación dorsal de la primera hilera del carpo que incrementa la carga por unidad de superficie a nivel de esta articulación del $50 \%$ en básculas dorsales de $20^{\circ}$ y alcanza el $67 \%$ con deformidades dorsales de $45^{\circ}{ }^{\circ}$. Así mismo se genera un cambio del centro de rotación de la muñeca que influye en la traslación normal de los tendones y se reduce su brazo de palanca, provocando una pérdida de fuerza del 50-60\% con respecto a la muñeca contralatera|8, ${ }^{8}$.

La consolidación en mala posición asocia acortamiento del radio y genera una discrepancia a nivel de la articulación radiocubital distal. En condiciones normales, sobre la articulación cúbitocarpiana se localizan el 17\% de las cargas axiales que actúan sobre la muñecal ${ }^{18}$. El alargamiento del cúbito con respecto al radio genera un incremento adicional, alcanzando el $42 \%$ de las cargas axiales cuando se asocian deformidades con varianzas positivas de $2.5 \mathrm{~mm}^{19}$. La incongruencia generada influye negativamente en el rango de movilidad de la articulación radiocubital distal, pudiendo ocasionar una reducción del arco de rotación del antebrazo de $47 \%$ de la pronación y $29 \%$ de la supinación ${ }^{20}$.

La necesidad de corrección de la deformidad en fracturas de la extremidad distal del radio que presentan una consolidación en mala posición sintomática no es un concepto nuevo ni reciente. En el año 1932, Ghormley y $\mathrm{Mroz}^{21}$ describieron la corrección quirúrgica de una deformidad del radio y en 1946 Speed y Knight ${ }^{22}$ presentaron el tratamiento de la consolidación en mala posición del radio distal mediante injerto de cresta iliaca y fijación interna. No obstante, la técnica de referencia en los últimos años es la descrita por Fernández en la década de los años ochenta ${ }^{23}$.

Debido al conocimiento que las fracturas inestables pueden desplazarse y al desarrollo de nuevos sistemas de fijación más fiables para el radio distal, se ha incrementado el tratamiento quirúrgico de las fracturas del radio distal, disminuyendo así la incidencia de consolidación en mala posición.

Nuestra serie muestra que la mayoría de los pacientes con consolidación en mala posición han sido tratados previamente de manera ortopédica, lo que coincide con la literatura consultada ${ }^{24,27}$. De los 23 pacientes solo 5 fueran sometidos a cirugía previamente y ninguno fue con algún sistema de fijación rígido.

El desplazamiento de los fragmentos en las fracturas del radio distal y su posterior consolidación en mala posición puede ocurrir en los tres ejes del espacio: sagital, originando una angulación dorsal o volar (más infrecuente); coronal o frontal, por el colapso de la columna radial habitualmente; y axial, por rotación del 


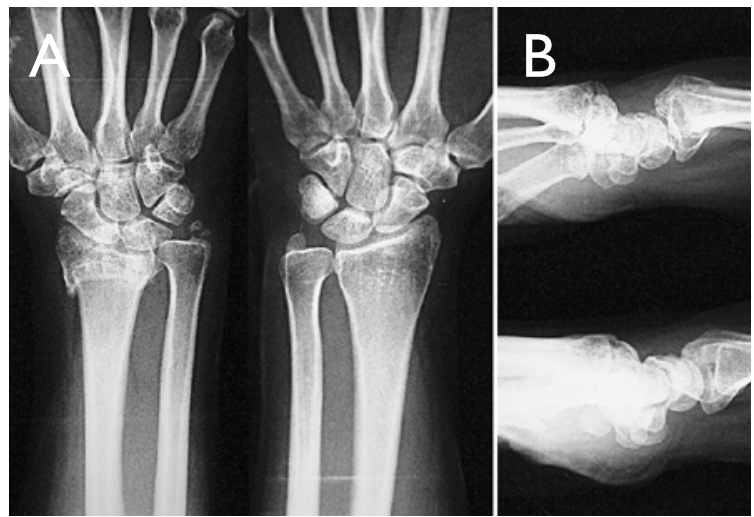

Figura 6. Imagen radiológica de una consolidación en acortamiento y báscula volar excesiva del radio distal comparado con la muñeca contralateral. Proyecciones posteroanterior A. y lateral B.

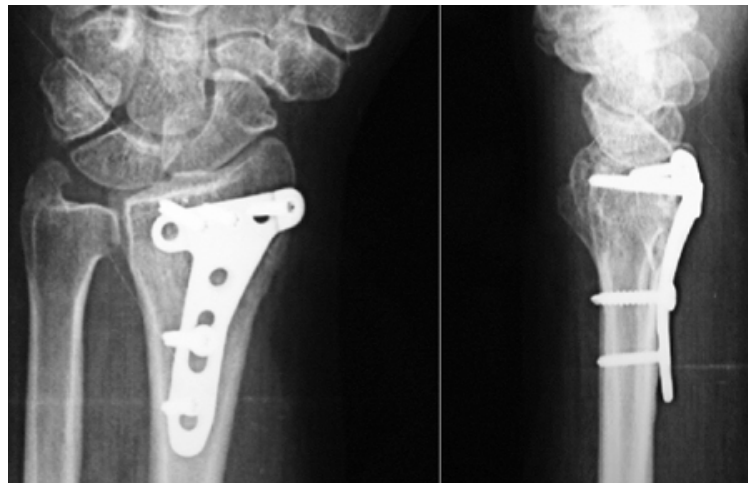

Figura 7. Control radiológico tras osteotomía de adición volar y fijación con placa y tornillos.

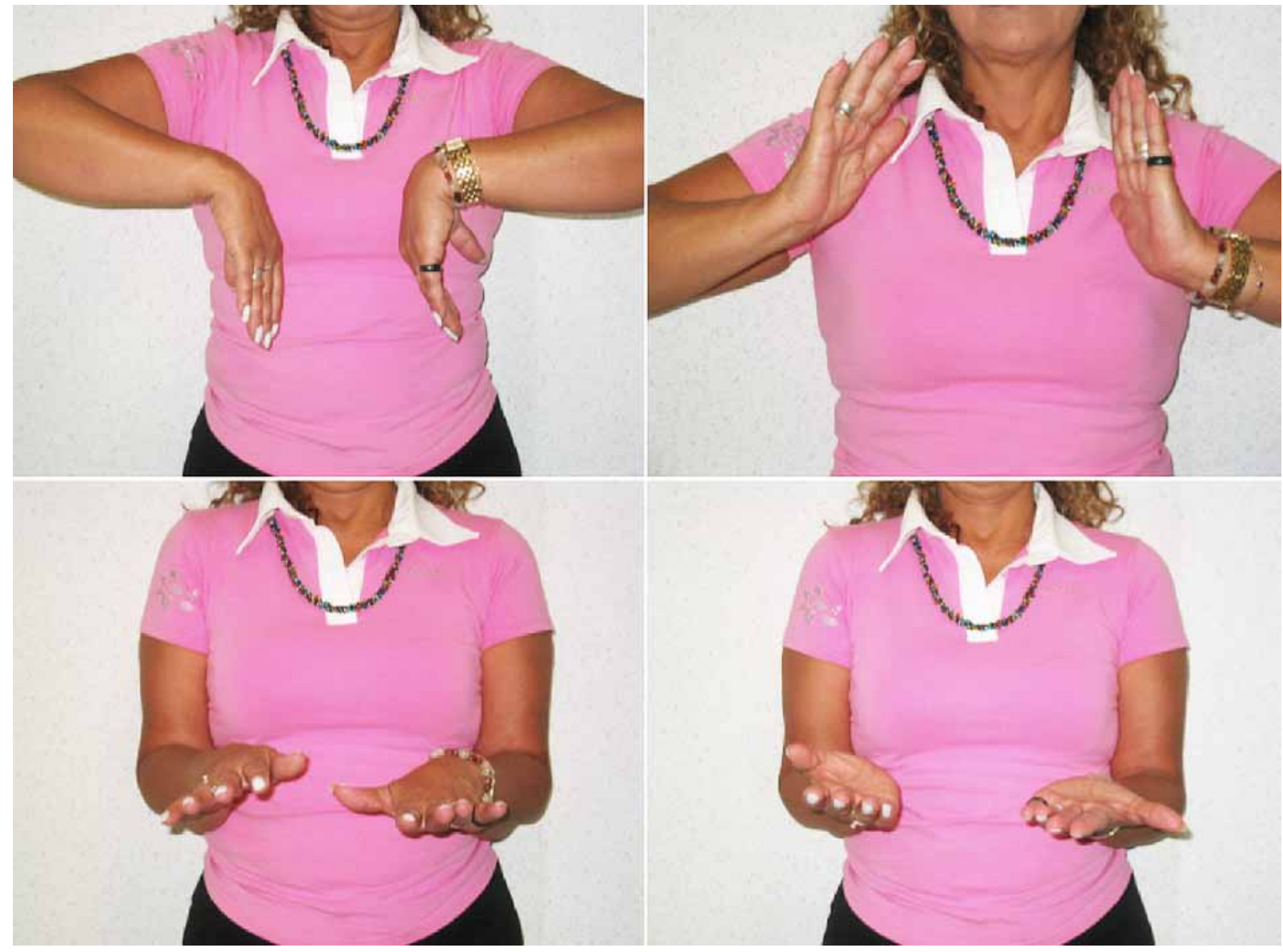

Figura 8. Arco de movilidad activo al año de la osteotomía correctora volar de adición.

fragmento distal en supinación en fracturas con angulación dorsal o pronación si la angulación es volaŕ Así como escalones articulares mayores de $2 \mathrm{~mm}$ son considerados como entidades preartrósicas, una angu- lación sagital del radio distal por encima de $20^{\circ}$ o un acortamiento superior a $4 \mathrm{~mm}$ también condicionan procesos degenerativos de la articulación y deben ser tratadas de forma quirúrgica en paciente jóvenes ${ }^{2,6}$. 
Si bien Fernández sugiere que los pacientes tratados quirúrgicamente con osteotomía del radio distal deben asociar un procedimiento de rescate de la articulación radiocubital distal ${ }^{23}$, los autores opinamos que una alineación correcta del radio distal durante la osteotomía no deja secuelas en la articulación radiocubital distal. De la serie presentada, solo un caso presentó una inestabilidad clínica en el periodo postoperatorio tardío que precisó un procedimiento de salvación (Darrach). Por esa misma razón, no indicamos la apertura del túnel carpiano para los pacientes con síntomas compresivos producidos por la deformidad del radio distal. Igualmente, el adecuado posicionamiento óseo soluciona los síntomas y no fue preciso su liberación.

La utilización del abordaje volar para la realización de la osteotomía permite la liberación del músculo pronator quadratus (PQ) que muchas veces está acortado por la cicatrización tras las fracturas del radio distal ${ }^{28}$. Esta razón puede explicar que la recuperación de la supinación fue mayor en el grupo de pacientes tratados con osteotomía por vía volar.

A diferencia de otras series revisadas ${ }^{24,27}$ que muestran grupos heterogéneos de pacientes (adultos jóvenes mezclados con mayores) y diferentes características de deformidad (intra o extra articular), nuestro grupo de pacientes fue lo más homogéneo posible, constituido por paciente jóvenes con deformidades extra-articulares del radio distal y con alta demanda funcional. Los resultados son similares a los publicados en series previas que muestran cómo el sistema de fijación dorsal para el radio distal presenta una incidencia mayor de complicaciones cuando se compara con la fijación por vía volar. Esta incidencia aumenta la necesidad de un segundo procedimiento quirúrgico para la retirada del material de osteosíntesis ${ }^{29}$. Recientemente se ha descrito una nueva técnica para la realización de correcciones de la deformidad dorsal del radio a partir de un abordaje volar convencional o ampliado a flexor carpi radialis ${ }^{24,30}$. Se fija el extremo distal del radio mediante un implante bloqueado de ángulo fijo y se realiza la osteotomía en la metáfisis distal del radio. Utilizado a modo de palanca se corrige la deformidad a través de la placa que se fija seguidamente una vez conseguida en la diáfisis radial. A través del mismo abordaje se introduce injerto de hueso esponjoso compactado que rellena el defecto óseo creado. De esta forma se evita la necesidad de un segundo abordaje para colocar el injerto óseo y la fijación con implantes dorsales que pueden precisar se retirados en un segundo tiempo.
La utilización de sistemas de fijación interna para las osteotomías con placas con tornillos de bloqueo, donde los tornillos distales se fijan a la placa, reducen el riesgo de desplazamiento óseo en el post-operatorio y precisan un tiempo menor de inmovilización. Sistemas convencionales de fijación, tipo consola, en muchas ocasiones no son capaces de soportar las cargas y necesitan de otros métodos de fijación asociados, como por ejemplo, un fijador externo ${ }^{31}$.

En todos los casos de osteotomías volares se utilizaron implantes de ángulo fijo. Estamos de acuerdo con Von Campe et al ${ }^{32}$ que describieron que, con el uso de sistemas de fijación de ángulo fijo, se puede hacer pequeños cambios de angulación durante la cirugía, a diferencia de otros métodos más tradicionales que precisan una planificación preoperatoria y un tallado del injerto muy precisos para llevar a cabo la osteotomía correctora.

En todas las osteotomías dorsales, la necesidad de alargamiento del radio fue inferior a $12 \mathrm{~mm}$, por lo que fue realizada una osteotomía incompleta por osteoclasia de la cortical volar y cubital del radio, que facilita la estabilización y permite la adición de injerto óseo esponjoso. En todos los casos fue utilizado injerto procedente de la cresta ilíaca y no fue necesaria la utilización de sustitutivos óseos y en contra de lo publicado por Arrington et $\mathrm{a}^{13}$, no se ha visto incrementado la morbilidad asociada a la toma de injerto y la incisión extra relacionada con la cresta iliaca.

En nuestra experiencia, en casos de larga evolución estamos de acuerdo con la necesidad de liberación o alargamiento del tendón del músculo braquiorradial así como la liberación del periostio dorsal del radio para facilitar en estos casos la corrección ósea ${ }^{28}$.

Todos nuestros casos precisaron una osteotomía de corrección mediante cuña de apertura. Creemos que la técnica con cuña de cierre asociada al acortamiento del cúbito debe ser utilizada solo en pacientes mayores, con baja demanda funcional, o contraindicación para extraer injerto óseo de la cresta iliaca, por la potencial pérdida de fuerza muscular por el acortamiento del antebrazo y las posibles complicaciones derivadas de un segundo sistema de fijación para el cúbito ${ }^{4}$.

En la serie analizada la osteotomía fue realizada a los 6 meses de media de la fractura y los resultados obtenidos (días de baja, reincorporación laboral, mejoría del dolor, parámetros radiológicos y calidad de vida) con ambas técnicas de osteotomía (dorsal y volar) fue- 
ron similares a las series publicadas $8,9,16,23,27$. En nuestra experiencia, la osteotomía dorsal presenta una mejor ganancia del arco de movilidad y la volar menos complicaciones y reintervenciones.

Estos resultados son acordes con lo propuesto por Jupiter y Ring ${ }^{34}$, que demostraron que la realización de la osteotomía de manera precoz facilita el procedimiento quirúrgico (osteotomía través de un tejido óseo inmaduro, con menor retracción de las partes blandas y menor inestabilidad de la articulación radiocubital distal) y disminuye el tiempo de baja laboral. Por esta razón, pacientes jóvenes con angulación dorsal mayor de 20 grados, deben ser considerados con una situación preartrósica y debe indicarse el procedimiento reconstructivo.

No obstante, este estudio presenta limitaciones. Se trata de un grupo pequeño de pacientes que no es suficiente para extraer conclusiones entre ambas opciones de osteotomía de corrección (dorsal o volar), si bien el objetivo inicial no era realizar un estudio comparativo sino valorar los resultados clínicos y laborales de los pacientes tratados en global y la muestra fue adecuada para este fin. Otro factor limitante es el tiempo de seguimiento medio (27 meses), que resulta corto para elaborar conclusiones a largo plazo, pero que cumplió el objetivo de valorar la reincorporación laboral a medio plazo.

Como conclusión, podemos afirmar que el tratamiento de la consolidación en mala posición del radio distal mediante osteotomías correctoras extraarticulares por adicción (volar o dorsal) ofrecen resultados clínicos, radiológicos y laborales satisfactorios en pacientes jóvenes sintomáticos, como la serie presentada. No obstante, la evolución a largo plazo sigue siendo una incógnita, por lo que precisamos estudios más largos para confirmar estos resultados.

\section{BIBLIOGRAFIA}

I. Colles A. On fracture of the carpal extremity of the radius. Edinb Med Surg Journal 1814;10:181315.

2. Cooney WP, Dobyns JH, Linscheid RL. Complications of Colles' fractures. J Bone Joint Surg. 1980;62A:6।3-19.

3. De Pedro JA, Blanco J. De Cabo A, García de Lucas F, Martin AP, Persson I, Ferreira J, Badia A. Resulta- dos del tratamiento quirúrgico de las fracturas del radio distal. Rev Ortop Traumatol. 2004;48:83-7.

4. Fernandez DL. Correction of posttraumatic wrist deformity in adults by osteotomy, bonegrafting and internal fixation. J Bone Joint Surg. 1982;64A: I 164-78.

5. Knirk J, Jupiter. Intra-articular fractures of the distal end of the radius in young adults. J Bone Joint Surg. 1986:68A:647-59.

6. González del Pino J, Bartolomé del Valle E, LópezGraña G, Ferreira-Villanova J. Consolidaciones viciosas tras fracturas del extremo distal del radio: patogenia, indicaciones y técnicas quirúrgicas. Rev Ortop Traumatol. 2003;47(supl. I):55-69.

7. Earnshaw SA, Aladin A, Surendran S, Moran CG. Closed reduction of Colles fractures: comparision of manual manipulation and finger trap-traction: a prospective, randomised study. J Bone Joint Surg. 2002;84A;354-8.

8. Prommersberger KJ, Van Schoonhoven J, Lanz UB. Outcome after corrective osteotomy for malunited fractures of the distal end of the radius. J Hand Surg. 2002;27B:55-60.

9. Fernandez DL. Radial osteotomy and Bowers arthroplasty for malunited fractures of the distal end of the radius. J Bone Joint Surg. 1988;70A: I538-5 I.

10. Park MJ, CooneyWP, Hahn ME, Looi KP,An K-N.The effects of dorsally angulated distal radius fractures on carpal kinematics. J Hand Surg. 2002;27A:22332.

I I Prommersberger KJ, Lanz U. Corrective osteotomy for malunited Colles fractures. Orthop Traumatol. 1998:6:75-87.

12. Fernández, DL, Jupiter JB. Fractures of the distal radius. A practical approach to management. New York: Springer-Verlag, 1995.

13. Castaing J. Les fractures récentes de l'extremité inférieure du radius chez l'adulte. Rev Chir Orthop. 1964:50:581-696.

14. Gartland JJ, Werley CW. Evaluation of healed Colles' fractures. J Bone Joint Surg. 195 I;33-A:895907.

15. Hevia-Campomanes E, Miranda F, Vivanco MC, Gomez F. Los accidentes de trabajo y las enfermedades profesionales. Gestión, prestaciones, procedimiento y jurisprudencia. Madrid: Colex, 1990.

16. Shea K, Fernandez DL, Júpiter JB, Martin C. Corrective osteotomy for malunited, volarly displaced fractures of the distal end of the radius. J Bone Joint Surg. 1997;79A: I 816-26. 
17. Rosales RS, Delgado EB, Díez de la Lastra-Bosch I. Evaluation of the Spanish version of the DASH and carpal tunnel syndrome health-related quality-oflife instruments: cross-cultural adaptation process y reliability. J Hand Surg. 2002; 27A:334-43.

I8. Iwasaki N, Minami A, Miyazawa T, Kaneda K. Force distribution through the wrist joint in patients with different stages of Kienböck disease: Using computed tomography osteoabsorptiometry. J Hand Surg. 2000; 28A: 870-6.

19. Werner FW, Palmer AK, Fortino MD, Short WH. Force transmisión through the distal ulna: effect of ulnar variance, lunate fossa angulation and radial and palmar tilt of the distal radius. J Hand Surg. 1992; 17A:423-8.

20. Bronstein AJ, Trumble TE, Tencer AF. The effects of distal radius fracture management on forearm rotation: a cadaveric study. J Hand Surg. 1997;22A:25862.

21. Ghormley RK, Mroz RJ. Fractures of the wrist. A review of one hundred seventy-six cases. Surg Gynec Obstet. 1932:57:377-81.

22. Speed JS, Knight RA. The treatment of malunited Colles's fractures.J Bone Joint Surg. 1945;27:36 I-7.

23. Fernandez DL. Correction of posttraumatic wrist deformity in adults by osteotomy, bone-grafting and internal fixation. J Bone Joint Surg. 1988;70A: 153851.

24. Sato K, Nakamura T, Iwamoto T, Toyama Y, Ikegami H, Takayama S. Corrective osteotomy for volarly malunited distal radius fracture. J Hand Surg. 2009;34-A:27-33).

25. Haase SC, Chung KC. Management of malunion of the distal radius. Hand Clin. 20 2;28:207-216.
26. Slagel BE, Luenam S, Pichora DR. Management of postraumatic malunion of fractures of the distal radius. Hand Clin. 20 1 0;26:71-84.

27. Buijze GA, Prommersberger KJ, Gonzalez del Pino J, Fernandez DL, Jupiter JB. Corrective osteotomy for combined intra and extra articular distal radius malunion. J Hand Surg. 20 12;37:204 I-9.

28. Orbay J, Badia A, Khoury R, Gonzales E, Indriago I. Volar fixed-angle fixacion of distal radius fracture:The DVR plate. Tech Hand Upper Extr Surg. 2004:8: 142-8.

29. Kambouroglou GK, Axelrod TS. Complications of the AO/ASIF titanium distal radius plate system ( $\pi$ plate) in internal fixation of the distal radius: a brief report. J Hand Surg. 1998;23A:737-4I.

30. Prommersberger K-J, Lanz UB. Corrective osteotomy of the distal radius through volar approach. Tech Hand Upper Extr Surg. 2004:8:70-7.

31. Ring D, Roberge C, Morgan T, Jupiter J. Osteotomy for malunited fractures of the distal radius: a comparison of structural and non-structural autogenous bone graf.J Hand Surg. 2002;27A:21 6-22.

32. von Campe A, Nagy L, Darius A, Dumond C. Corrective osteotomies in malunions of the distal radius. Do we get what we planned?. Clin Orthop. 2006:450:179-85.

33. Arrington ED, Smith WJ, Chambers H, Bucknell AL, Davino NA. Complications of iliac crest bone graft harvesting. Clin Orthop. 1996; 329:330-9.

34. Jupiter JB, Ring D. A comparison of early and late reconstruction of malunited fractures of the distal end of the radius. J Bone Joint Surg. 1996;78A:739-48. 